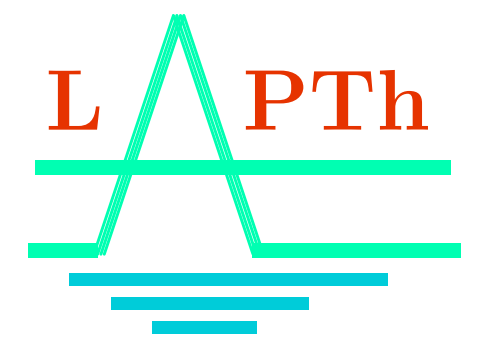

Laboratoire d'Annecy-leVieux de Physique Théorique

website: http://lappweb.in2p3.fr/lapth-2005/

\title{
Algebraic approach to multiple defects on the line and application to Casimir force
}

\author{
M. Mintchev回 and E. Ragoucy ${ }^{2}$ \\ ${ }^{a}$ INFN and Dipartimento di Fisica, Università di Pisa, Largo Pontecorvo 3, 56127 \\ Pisa, Italy \\ ${ }^{b}$ LAPTH, 9, Chemin de Bellevue, BP 110, F-74941 Annecy-le-Vieux cedex, France
}

\begin{abstract}
An algebraic framework for quantization in presence of arbitrary number of point-like defects on the line is developed. We consider a scalar field which interacts with the defects and freely propagates away of them. As an application we compute the Casimir force both at zero and finite temperature. We derive also the charge density in the Gibbs state of a complex scalar field with defects. The example of two delta-defects is treated in detail.
\end{abstract}

IFUP-TH 07/2007

LAPTH-1177/07

hep-th/yymm.nnnn

\footnotetext{
${ }^{1}$ mintchev@df.unipi.it

${ }^{2}$ ragoucy@lapp.in2p3.fr
} 


\section{Introduction}

Quantum fields with impurities (defects) are recently subject of intensive studies in the context of condensed matter physics [1]- [5], conformal field theory $[6,7]$ and integrable systems [8]- [16]. Most of the papers concern the case of one point-like defect. For more realistic applications however, the generalization to $n>1$ defects would be essential. In the present paper we face the multi-defect problem by extending the algebraic technique developed in [14]- [18] for $n=1$. We will show below that this technique perfectly applies to the case $n>1$ as well, the key point being the reformulation of the boundary value problem at hand in algebraic terms.

An immediate application of this formalism is the derivation of the Casimir force acting on a test particle. Since this issue is of some experimental interest, we treat it in detail, deriving the general expression of force. We consider also a complex scalar field and determine the relative charge density. As an explicit example we focus on two delta-type defects. It appears that the intensity and direction of the force depend on the position. The force is discontinuous at the defects and actually diverges when one is approaching any of them from the left (right). A compensation occurs however in the symmetric limit, which is finite.

The paper is organized as follows. Introducing the notation, in the next section we briefly summarize the algebraic approach to one defect. This approach is generalized in section [3, where the solution for two defects is established. The latter is extended to $n>2$ defects in section 4 . In section 5 we establish the two-point correlation function both at zero and finite temperature. Using this results we compute the Casimir force (section 5.2) and the charge density of the complex scalar field (section 5.4). In section 6 we consider in details two delta-defects and discover a kind of resonance effect in the behavior of the density between the two defects. Some remarks and our conclusions are collected in section 7 .

\section{One defect: reflection-transmission algebra}

It is useful to recall first the algebraic treatment [15] of one defect on the real line. We take a defect with arbitrary position $y \in \mathbb{R}$. It divides the real line in two domains $D_{1}=\{x \in \mathbb{R}: x<y\}$ and $D_{2}=\{x \in \mathbb{R}: x>y\}$. For simplicity we focus on the real massive scalar field $\varphi(t, x)$, satisfying

$$
\left[\partial_{t}^{2}-\partial_{x}^{2}+m^{2}\right] \varphi(t, x)=0, \quad x \neq y .
$$

It is well known [19] that all possible dissipationless interactions of $\varphi(t, x)$ with the defect $y$ are described by the boundary condition

$$
\left(\begin{array}{c}
\varphi(t,+y) \\
\partial_{x} \varphi(t,+y)
\end{array}\right)=\left(\begin{array}{cc}
a & b \\
c & d
\end{array}\right)\left(\begin{array}{c}
\varphi(t,-y) \\
\partial_{x} \varphi(t,-y)
\end{array}\right), \quad \forall t \in \mathbb{R},
$$


where

$$
a d-b c=1, \quad a, \ldots, d \in \mathbb{R} .
$$

Following [17,18], one can solve equations (2.1|2.2) by introducing the so called reflectiontransmission algebra with the following structure. With each domain $D_{i}$ one associates a "creation" and "annihilation" operator $\left\{a_{i}^{*}(k), a_{i}(k): k \in \mathbb{R}\right\}$ which satisfy

$$
\begin{aligned}
& {\left[a_{i}(k), a_{j}(p)\right]=0=\left[a_{i}^{*}(k), a_{j}^{*}(p)\right],} \\
& {\left[a_{i}(k), a_{j}^{*}(p)\right]=\left[\delta_{i j}+T_{i j}(k)\right] 2 \pi \delta(k-p)+R_{i j}(k) 2 \pi \delta(k+p),}
\end{aligned}
$$

where

$$
T(k)=\left(\begin{array}{cc}
0 & T_{12}(k) \\
T_{21}(k) & 0
\end{array}\right), \quad R(k)=\left(\begin{array}{cc}
R_{11}(k) & 0 \\
0 & R_{22}(k)
\end{array}\right),
$$

represent the reflection and transmission coefficients from the impurity. In terms of the parameters $\{a, \ldots, d\}$ one has

$$
\begin{aligned}
R_{11}(k) & =\frac{b k^{2}+\mathrm{i}(a-d) k+c}{b k^{2}+\mathrm{i}(a+d) k-c} \mathrm{e}^{-2 \mathrm{i} k y}, & T_{12}(k) & =\frac{2 \mathrm{i} k}{b k^{2}+\mathrm{i}(a+d) k-c}, \\
T_{21}(k) & =\frac{-2 \mathrm{i} k}{b k^{2}-\mathrm{i}(a+d) k-c}, & R_{22}(k) & =\frac{b k^{2}+\mathrm{i}(a-d) k+c}{b k^{2}-\mathrm{i}(a+d) k-c} \mathrm{e}^{-2 \mathrm{i} k y} .
\end{aligned}
$$

Let us stress that the reflection coefficients (but not the transmission ones) depend on the position $y$ of the impurity: in the following, we will also use the 'bare' coefficients $\widetilde{R}_{j j}(k)$ which are just the part independent from the position $y$ :

$$
R_{11}(k)=\widetilde{R}_{11}(k) \mathrm{e}^{-2 \mathrm{i} k y}, \quad R_{22}(k)=\widetilde{R}_{22}(k) \mathrm{e}^{-2 \mathrm{i} k y} .
$$

The associated defect scattering matrix

$$
S(k)=T(k)+R(k)
$$

satisfies unitarity and Hermitian analyticity

$$
S(k) S(k)^{\dagger}=\mathbb{I}, \quad S(k)^{\dagger}=S(-k) .
$$

Now, the solution of (2.1) can be written in the form [17]

$$
\left.\varphi(t, x)\right|_{x \in D_{i}} \equiv \varphi_{i}(t, x)=\int_{-\infty}^{+\infty} \frac{\mathrm{d} k}{2 \pi \sqrt{2 \omega(k)}}\left[a_{i}^{*}(k) \mathrm{e}^{\mathrm{i} \omega(k) t-\mathrm{i} k x}+a_{i}(k) \mathrm{e}^{-\mathrm{i} \omega(k) t+\mathrm{i} k x}\right] .
$$

where $\omega(k)=\sqrt{k^{2}+m^{2}}$. The boundary condition (2.2) then implies that $\left\{a_{1}^{*}(k), a_{1}(k)\right\}$ and $\left\{a_{2}^{*}(k), a_{2}(k)\right\}$ are not independent, but satisfy the constraints

$$
\begin{aligned}
& a_{i}(k)=\sum_{j=1}^{2}\left(R_{i j}(k) a_{j}(-k)+T_{i j}(k) a_{j}(k)\right), \\
& a_{i}^{*}(k)=\sum_{j=1}^{2}\left(a_{j}^{*}(-k) R_{j i}(-k)+a_{j}^{*}(k) T_{j i}(k)\right),
\end{aligned}
$$


which are consistent with (2.4) because of (2.10). The algebra $\mathfrak{A}_{1}$ defined by equations (2.4, 2.12, 2.13) is known as reflection-transmission (RT) algebra [15]. Introducing the matrices

$$
A(k) \equiv\left(\begin{array}{c}
a_{1}(k) \\
a_{2}(-k)
\end{array}\right), \quad C(k) \equiv\left(\begin{array}{cc}
R_{11}(k) & T_{12}(k) \\
T_{21}(-k) & R_{22}(-k)
\end{array}\right)
$$

equations (2.12, 2.13) can be conveniently written in the form

$$
A(k)=C(k) A(-k), \quad A(k)^{\dagger}=A(-k)^{\dagger} C(k)^{\dagger} .
$$

By construction the matrix $C(k)$ satisfies

$$
C(k) C(-k)=\mathbb{I}, \quad C(k)^{\dagger}=C(-k)^{t},
$$

where the apex $t$ denotes transposition.

We end this summary with two observations. In the domain of $\{a, \ldots, d\}$ where bound states are absent [17], the field $\varphi(t, x)$ satisfies in addition to (2.1, 2.2) the equal-time canonical commutation relations

$$
\left[\varphi\left(t, x_{1}\right), \varphi\left(t, x_{2}\right)\right]=0, \quad\left[\left(\partial_{t} \varphi\right)\left(t, x_{1}\right), \varphi\left(t, x_{2}\right)\right]=-i \delta\left(x_{1}-x_{2}\right) .
$$

We will not consider in this paper the issue of bound states, referring for the details about their impact to [17].

We stress in conclusion that the above construction is purely algebraic and applies for any representation of the RT algebra $\mathfrak{A}_{1}$. This fact allows to investigate the physical effects associated with nonequivalent representations and in particular, to introduce [17] the concept of temperature, which is relevant for the applications in section 5.

\section{The RT algebra of two defects}

We show now how the RT algebraic framework allows to compute in a very systematic and straightforward way the global reflection and transmission coefficients when there are two defects. We confirm the results of [20], where these coefficients where computed through the calculation of Green functions.

We consider two defects located at $y_{1}<y_{2}$. At each of them

$$
\left(\begin{array}{c}
\varphi\left(t,+y_{\alpha}\right) \\
\partial_{x} \varphi\left(t,+y_{\alpha}\right)
\end{array}\right)=\left(\begin{array}{cc}
a_{\alpha} & b_{\alpha} \\
c_{\alpha} & d_{\alpha}
\end{array}\right)\left(\begin{array}{c}
\varphi\left(t,-y_{\alpha}\right) \\
\partial_{x} \varphi\left(t,-y_{\alpha}\right)
\end{array}\right), \quad \alpha=1,2 .
$$

Let $R^{[\alpha]}(k)$ and $T^{[\alpha]}(k)$ be the reflection and transition matrices defined by (2.7) in terms of the parameters $\left\{a_{\alpha}, \ldots, d_{\alpha}\right\}$. According to (2.14) we set

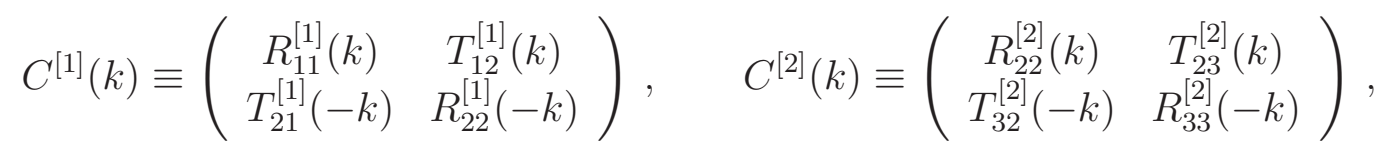


which satisfy by construction

$$
C^{[\alpha]}(k) C^{[\alpha]}(-k)=\mathbb{I}, \quad C^{[\alpha]}(k)^{\dagger}=C^{[\alpha]}(-k)^{t}, \quad \alpha=1,2 .
$$

The two defects separate now the real line in three domains

$$
D_{1}=\left\{x \in \mathbb{R}: x<y_{1}\right\}, \quad D_{2}=\left\{x \in \mathbb{R}: y_{1}<x<y_{2}\right\}, \quad D_{3}=\left\{x \in \mathbb{R}: y_{2}<x\right\} \text {. }
$$

Following the previous section, we associate with each of them the operators $\left\{a_{i}^{*}(k), a_{i}(k)\right\}$ and define the field $\varphi(t, x)$ by (2.11), where now $i=1,2,3$. Since $\varphi(t, x)$ must satisfy (3.1), we impose

$$
A^{[\alpha]}(k)=C^{[\alpha]}(k) A^{[\alpha]}(-k), \quad A^{[\alpha]}(k)^{\dagger}=A^{[\alpha]}(-k)^{\dagger} C^{[\alpha]}(k)^{\dagger}, \quad \alpha=1,2,
$$

where

$$
A^{[\alpha]}(k) \equiv\left(\begin{array}{c}
a_{\alpha}(k) \\
a_{\alpha+1}(-k)
\end{array}\right), \quad \alpha=1,2 .
$$

The operators $\left\{a_{i}^{*}(k), a_{i}(k)\right\}$ describe the propagation of $\varphi(t, x)$ in $D_{i}$. Only $\left\{a_{1}^{*}(k), a_{1}(k)\right\}$ and $\left\{a_{3}^{*}(k), a_{3}(k)\right\}$ give origin to asymptotic states. Therefore it is natural to expect that $\left\{a_{2}^{*}(k), a_{2}(k)\right\}$ can be expressed in terms of $\left\{a_{1}^{*}(k), a_{1}(k)\right\}$ and $\left\{a_{3}^{*}(k), a_{3}(k)\right\}$. Indeed, from (3.5) it follows that

$$
\begin{aligned}
C_{11}^{[2]}(k) a_{2}(-k) & =a_{2}(k)-C_{12}^{[2]}(k) a_{3}(k), \\
C_{22}^{[1]}(-k) a_{2}(-k) & =a_{2}(k)-C_{21}^{[1]}(-k) a_{1}(k) .
\end{aligned}
$$

Eliminating $a_{2}(-k)$ from these two equations, one gets

$$
\left[C_{11}^{[2]}(k)-C_{22}^{[1]}(-k)\right] a_{2}(k)=C_{11}^{[2]}(k) C_{21}^{[1]}(-k) a_{1}(k)-C_{22}^{[1]}(-k) C_{12}^{[2]}(k) a_{3}(k) .
$$

Remark that because of the factors $\mathrm{e}^{2 \mathrm{i} k y_{2}}$ and $\mathrm{e}^{2 \mathrm{i} k y_{1}}$ appearing in $R_{22}^{[2]}(k)$ and $R_{22}^{[1]}(k)$ respectively, the coefficient $C_{11}^{[2]}(k)-C_{22}^{[1]}(-k)$ never vanishes for generic $y_{1}, y_{2}$ and (3.8) uniquely determines $a_{2}(k)$ in terms of $a_{1}(k)$ and $a_{3}(k)$. One has

$$
a_{2}(k)=\frac{R_{22}^{[2]}(k) T_{21}^{[1]}(-k) a_{1}(k)-R_{22}^{[1]}(k) T_{23}^{[2]}(k) a_{3}(k)}{R_{22}^{[2]}(k)-R_{22}^{[1]}(k)},
$$

Analogously, $a_{2}^{*}(k)$ is given in terms of $a_{1}^{*}(k)$ and $a_{3}^{*}(k)$,

$$
a_{2}^{*}(k)=\frac{R_{22}^{[2]}(-k) T_{12}^{[1]}(-k) a_{1}^{*}(k)-R_{22}^{[1]}(-k) T_{32}^{[2]}(k) a_{3}^{*}(k)}{R_{22}^{[2]}(-k)-R_{22}^{[1]}(-k)},
$$


We conclude therefore that in order to define the RT algebra for two impurities $\mathfrak{A}_{2}$, it is enough to fix the commutation relations between $\left\{a_{1}^{*}(k), a_{1}(k)\right\}$ and $\left\{a_{3}^{*}(k), a_{3}(k)\right\}$. For this purpose we have to determine the global reflection and transmission matrices

$$
\mathcal{T}(k)=\left(\begin{array}{cc}
0 & \mathcal{T}_{13}(k) \\
\mathcal{T}_{31}(k) & 0
\end{array}\right), \quad \mathcal{R}(k)=\left(\begin{array}{cc}
\mathcal{R}_{11}(k) & 0 \\
0 & \mathcal{R}_{33}(k)
\end{array}\right)
$$

in terms of the local ones $R^{[\alpha]}$ and $T^{[\alpha]}$. According to (2.12), $\mathcal{T}(k)$ and $\mathcal{R}(k)$ can be read from the relations between $a_{1}(k)$ and $a_{3}(k)$, which can be derived as follows. Plugging the expression (3.8) into (3.5), we get

$$
a_{1}(k)=C_{22}^{[1]}(-k)^{-1} C_{11}^{[1]}(k) \frac{C_{22}^{[1]}(-k)-C_{11}^{[2]}(k)}{1-C_{22}^{[1]}(k) C_{11}^{[2]}(k)} a_{1}(-k)+\frac{C_{12}^{[1]}(k) C_{12}^{[2]}(k)}{1-C_{22}^{[1]}(k) C_{11}^{[2]}(k)} a_{3}(k) .
$$

By means of the identities

$$
\begin{gathered}
C_{22}^{[1]}(-k)^{-1}=C_{22}^{[1]}(k)+C_{22}^{[1]}(-k)^{-1} C_{12}^{[1]}(k) C_{21}^{[1]}(-k), \\
C_{12}^{[1]}(-k) C_{22}^{[1]}(-k)^{-1}=-C_{11}^{[1]}(k)^{-1} C_{12}^{[1]}(k),
\end{gathered}
$$

following from (3.3), equation (3.12) can be rewritten in the form

$$
a_{1}(k)=\left[C_{11}^{[1]}(k)+\frac{C_{12}^{[1]}(k) C_{21}^{[1]}(k) C_{11}^{[2]}(k)}{1-C_{22}^{[1]}(k) C_{11}^{[2]}(k)}\right] a_{1}(-k)+\frac{C_{12}^{[1]}(k) C_{12}^{[2]}(k)}{1-C_{22}^{[1]}(k) C_{11}^{[2]}(k)} a_{3}(k) .
$$

The same type of calculations provides

$$
a_{3}(k)=\left[C_{22}^{[2]}(-k)+\frac{C_{21}^{[2]}(-k) C_{12}^{[2]}(-k) C_{22}^{[1]}(-k)}{1-C_{22}^{[1]}(-k) C_{11}^{[2]}(-k)}\right] a_{3}(-k)+\frac{C_{21}^{[2]}(-k) C_{21}^{[1]}(-k)}{1-C_{22}^{[1]}(-k) C_{11}^{[2]}(-k)} a_{1}(k) .
$$

Comparing (3.13, 3.14) with (2.12), one infers

$$
\begin{gathered}
\mathcal{R}_{11}(k)=C_{11}^{[1]}(k)+\frac{C_{12}^{[1]}(k) C_{21}^{[1]}(k) C_{11}^{[2]}(k)}{1-C_{22}^{[1]}(k) C_{11}^{[2]}(k)}:=\widetilde{\mathcal{R}}_{11}(k) \mathrm{e}^{-2 \mathrm{i} k y_{1}} \\
\widetilde{\mathcal{R}}_{11}(k)=\widetilde{R}_{11}^{[1]}(k)+\frac{T_{12}^{[1]}(k) T_{21}^{[1]}(-k) \widetilde{R}_{22}^{[2]}(k) \mathrm{e}^{2 \mathrm{i} k y_{12}}}{1-\widetilde{R}_{22}^{[1]}(-k) \widetilde{R}_{22}^{[2]}(k) \mathrm{e}^{2 \mathrm{i} k y_{12}}}, \\
\mathcal{R}_{33}(k)=C_{22}^{[2]}(-k)+\frac{C_{21}^{[2]}(-k) C_{12}^{[2]}(-k) C_{22}^{[1]}(-k)}{1-C_{22}^{[1]}(-k) C_{11}^{[2]}(-k)}:=\widetilde{\mathcal{R}}_{33}(k) \mathrm{e}^{-2 \mathrm{i} k y_{2}} \\
\widetilde{\mathcal{R}}_{33}(k)=\widetilde{R}_{33}^{[2]}(k)+\frac{T_{32}^{[2]}(k) T_{23}^{[2]}(-k) \widetilde{R}_{22}^{[1]}(k) \mathrm{e}^{-2 \mathrm{i} k y_{12}}}{1-\widetilde{R}_{22}^{[1]}(k) \widetilde{R}_{22}^{[2]}(-k) \mathrm{e}^{-2 \mathrm{i} k y_{12}}},
\end{gathered}
$$




$$
\begin{gathered}
\mathcal{T}_{13}(k)=\frac{C_{12}^{[1]}(k) C_{12}^{[2]}(k)}{1-C_{22}^{[1]}(k) C_{11}^{[2]}(k)}=\frac{T_{12}^{[1]}(k) T_{23}^{[2]}(k)}{1-\widetilde{R}_{22}^{[1]}(-k) \widetilde{R}_{22}^{[2]}(k) \mathrm{e}^{2 \mathrm{i} k y_{12}}}, \\
\mathcal{T}_{31}(k)=\frac{C_{21}^{[2]}(-k) C_{21}^{[1]}(-k)}{1-C_{22}^{[1]}(-k) C_{11}^{[2]}(-k)}=\frac{T_{32}^{[2]}(k) T_{21}^{[1]}(k)}{1-\widetilde{R}_{22}^{[1]}(k) \widetilde{R}_{22}^{[2]}(-k) \mathrm{e}^{-2 \mathrm{i} k y_{12}}},
\end{gathered}
$$

which express the global two-impurity reflection and transmission coefficients in terms of the local ones. As for the one-impurity case, we have introduced 'bare' reflection coefficients which, as the transmission coefficients, depend only on the distance between the two impurities, $y_{12}=y_{1}-y_{2}$.

Remark that when one of the two defect is 'trivial', i.e. $R_{11}(k)=R_{22}(k)=0$ and $T_{12}(k)=T_{21}(k)=1$, the global coefficients $\mathcal{R}$ and $\mathcal{T}$ become identical to the ones of the remaining defect.

The relative $S$-matrix is

$$
S(k)=\mathcal{T}(k)+\mathcal{R}(k) .
$$

Using the expressions $(3.17+3.20)$ and the properties of $R^{[\alpha]}(k)$ and $T^{[\alpha]}(k)$ one can verify that (3.21) satisfies both unitarity and Hermitian analyticity (2.10), which are fundamental for the construction of the RT algebra $\mathfrak{A}_{2}$ below.

Equations (3.17] 3.20) describe in a compact way all (possible infinite) processes of reflection and transmission from the two defects, relating an incoming to an outgoing wave. Accordingly, we impose on the $\mathfrak{A}_{2}$-generators

$$
\begin{aligned}
& {\left[a_{i}(k), a_{j}(p)\right]=0=\left[a_{i}^{*}(k), a_{j}^{*}(p)\right],} \\
& {\left[a_{i}(k), a_{j}^{*}(p)\right]=\left[\delta_{i j}+\mathcal{T}_{i j}(k)\right] 2 \pi \delta(k-p)+\mathcal{R}_{i j}(k) 2 \pi \delta(k+p),}
\end{aligned}
$$

where $i, j$ take only the values 1 and 3 . The exchange relations involving $\left\{a_{2}^{*}(k), a_{2}(k)\right\}$ are determined by (3.22) and the relations (3.9, 3.10).

Summarizing, the RT algebra $\mathfrak{A}_{2}$ for two defects has the following simple structure. The elements $\left\{a_{2}^{*}(k), a_{2}(k)\right\}$, associated with the field evolution between the defects, are linear combinations of $\left\{a_{1}^{*}(k), a_{1}(k)\right\}$ and $\left\{a_{3}^{*}(k), a_{3}(k)\right\}$, which generate the asymptotic states and satisfy quadratic exchange relations (3.22). The latter have the same form as in the one-impurity case (2.4), but involve the global two-impurity reflection and transmission matrices $\mathcal{R}(k)$ and $\mathcal{T}(k)$, which are uniquely determined by the single defects via (3.15) 3.20). We will show in the next section that this result has a straightforward generalization to an arbitrary number $n>2$ of defects. 


\section{General case of several defects}

We consider here $n$ defects on $\mathbb{R}$ with coordinates $y_{1}<\cdots<y_{n}$. They define the $n+1$ domains

$D_{1}=\left\{x \in \mathbb{R}: x<y_{1}\right\}, \ldots, D_{i}=\left\{x \in \mathbb{R}: y_{i-1}<x<y_{i}\right\}, \ldots, D_{n+1}=\left\{x \in \mathbb{R}: y_{n}<x\right\}$

The relative RT algebra $\mathfrak{A}_{n}$ is therefore generated by $\left\{a_{i}^{*}(k), a_{i}(k): i=1, \ldots, n+1\right\}$. Extrapolating from the previous section, we expect that all $\left\{a_{i}^{*}(k), a_{i}(k): i=2, \ldots, n\right\}$ can be expressed in terms of $\left\{a_{1}^{*}(k), a_{1}(k)\right\}$ and $\left\{a_{n+1}^{*}(k), a_{n+1}(k)\right\}$, which generate in turn the asymptotic states. Let us now verify this scenario and determine the algebra $\mathfrak{A}_{n}$. Setting

$$
C^{[\alpha]}(k) \equiv\left(\begin{array}{cc}
R_{\alpha \alpha}^{[\alpha]}(k) & T_{\alpha(\alpha+1)}^{[\alpha]}(k) \\
T_{(\alpha+1) \alpha}^{[\alpha]}(-k) & R_{(\alpha+1)(\alpha+1)}^{[\alpha]}(-k)
\end{array}\right), \quad \alpha=1, \ldots, n,
$$

we infer from the boundary condition (3.1) that (3.5, 3.9) hold now for any $\alpha=1, \ldots, n$.

Indeed, applying recursively the technics used in the case of two defects, one deduces that

$$
A^{[j, l]}(k)=\left(\begin{array}{c}
a_{j}(k) \\
a_{l}(-k)
\end{array}\right), \quad j, k=1, \ldots, n+1,
$$

obeys the relations

$$
\begin{aligned}
A^{[j, l]}(k) & =C^{[j \ldots l]}(k) A^{[j, l]}(-k) \\
C^{[j \ldots l]}(k)^{\dagger} & =C^{[j \ldots l]}(-k)^{t} \quad \text { and } \quad C^{[j \ldots l]}(k) C^{[j \ldots l]}(-k)=\mathbb{I}, \quad 1 \leq j<l \leq n+1
\end{aligned}
$$

where $C^{[j \ldots l]}(k)$ is some matrix built on the $C^{[\alpha]}(k)$ matrices, $\alpha=j, \ldots, l$. Starting from

$$
\begin{aligned}
& a_{j}(k)=C_{21}^{[1 \ldots j]}(k) a_{1}(k)+C_{22}^{[1 \ldots j]}(k) a_{j}(-k), \\
& a_{j}(k)=C_{11}^{[j \ldots n+1]}(k) a_{j}(-k)+C_{12}^{[j \ldots n+1]}(k) a_{n+1}(k),
\end{aligned}
$$

one gets

$$
\left(C_{11}^{[j \ldots n+1]}(k)-C_{22}^{[1 \ldots j]}(k)\right) a_{j}(k)=C_{21}^{[1 \ldots j]}(k) C_{11}^{[j \ldots n+1]}(k) a_{1}(k)-C_{22}^{[1 \ldots j]}(k) C_{12}^{[j \ldots n+1]}(k) a_{n+1}(k) .
$$

Therefore, the expressions found in the case of two defects also apply here, with the rules

$$
\begin{array}{r}
C^{[1]}(k) \rightarrow C^{[1 \ldots j]}(k) ; C^{[2]}(k) \rightarrow C^{[j \ldots n+1]}(k), \\
a_{1}(k) \rightarrow a_{1}(k) ; a_{2}(k) \rightarrow a_{j}(k) ; a_{3}(k) \rightarrow a_{n+1}(k) .
\end{array}
$$

Hence, it remains to compute the matrices $C^{[j \ldots l]}(k)$ from the matrices $C^{[\alpha]}(k)$. We give 
here a recursion formula for them. We consider the relations

$$
\begin{aligned}
A^{[1]}(k) & =C^{[1]}(k) A^{[1]}(-k), \\
C^{[1]}(k)^{\dagger} & =C^{[1]}(-k)^{t} \text { and } C^{[1]}(k) C^{[1]}(-k)=\mathbb{I}, \\
A^{[2, n+1]}(k) & =C^{[2 \ldots n+1]}(k) A^{[2, n+1]}(-k), \\
C^{[2 \ldots n+1]}(k)^{\dagger} & =C^{[2 \ldots n+1]}(-k)^{t} \text { and } C^{[2, n+1]}(k) C^{[2 \ldots n+1]}(-k)=\mathbb{I},
\end{aligned}
$$

which amounts to take $j=2$ in the previous calculations. This leads to

$$
A^{[1, n+1]}(k)=C^{[1 \ldots n+1]}(k) A^{[1, n+1]}(-k),
$$

or in components

$$
\begin{aligned}
a_{1}(k) & =C_{11}^{[1 \ldots n+1]}(k) a_{1}(-k)+C_{12}^{[1 \ldots n+1]}(k) a_{n+1}(-k), \\
a_{n+1}(k) & =C_{21}^{[1 \ldots n+1]}(k) a_{1}(-k)+C_{22}^{[1 \ldots n+1]}(k) a_{n+1}(-k),
\end{aligned}
$$

where

$$
\begin{aligned}
& C_{11}^{[1 \ldots n+1]}(k)=C_{11}^{[1]}(k)+\frac{C_{12}^{[1]}(k) C_{21}^{[1]}(k) C_{22}^{[2 \ldots n+1]}(k)}{1-C_{22}^{[1]}(k) C_{11}^{[2 \ldots n+1]}(k)}, \\
& C_{12}^{[1 \ldots n+1]}(k)=\frac{C_{12}^{[1]}(k) C_{12}^{[2 \ldots n+1]}(k)}{1-C_{22}^{[1]}(k) C_{11}^{[2 \ldots n+1]}(k)}, \\
& C_{21}^{[1 \ldots n+1]}(k)=\frac{C_{21}^{[2 \ldots n+1]}(k) C_{21}^{[1]}(k)}{1-C_{22}^{[1]}(k) C_{11}^{[2 \ldots n+1]}(k)}, \\
& C_{22}^{[1 \ldots n+1]}(k)=C_{22}^{[2 \ldots n+1]}(k)+\frac{C_{21}^{[2 \ldots n+1]}(k) C_{12}^{[2 \ldots n+1]}(k) C_{22}^{[1]}(k)}{1-C_{22}^{[1]}(k) C_{11}^{[2 \ldots n+1]}(-k)} .
\end{aligned}
$$

Hence, an iterative use of these formulae, starting from the 'local' coefficients $C^{[\alpha]}(k)$, allows to compute all the $C^{[j \ldots l]}(k)$.

Alternative recursion formulas can be obtained particularizing the last defect instead of the first one. One gets

$$
\begin{aligned}
& C_{11}^{[1 \ldots n+1]}(k)=C_{11}^{[1 \ldots n]}(k)+\frac{C_{12}^{[1 \ldots n]}(k) C_{21}^{[1 \ldots n]}(k) C_{22}^{[n+1]}(k)}{1-C_{22}^{[1 \ldots n]}(k) C_{11}^{[n+1]}(k)}, \\
& C_{12}^{[1 \ldots n+1]}(k)=\frac{C_{12}^{[1 \ldots n]}(k) C_{12}^{[n+1]}(k)}{1-C_{22}^{[1 \ldots n]}(k) C_{11}^{[n+1]}(k)}, \\
& C_{21}^{[1 \ldots n+1]}(k)=\frac{C_{21}^{[n+1]}(k) C_{21}^{[1 \ldots n]}(k)}{1-C_{22}^{[1 \ldots n]}(k) C_{11}^{[n+1]}(k)}, \\
& C_{22}^{[1 \ldots n+1]}(k)=C_{22}^{[n+1]}(k)+\frac{C_{21}^{[n+1]}(k) C_{12}^{[n+1]}(k) C_{22}^{[1 \ldots n]}(k)}{1-C_{22}^{[1 \ldots n]}(k) C_{11}^{[n+1]}(-k)} .
\end{aligned}
$$


Note also that one can deduce physical properties directly from the recursion formulae. In particular, from unitarity and hermiticity relations of $C^{[1]}(k)$ and $C^{[2 \ldots n+1]}(k)$, it is easy to check that these relations are also obeyed by $C^{[1 \ldots n+1]}(k)$ :

$$
C^{[1 \ldots n+1]}(k) C^{[1 \ldots n+1]}(-k)=\mathbb{I} \quad \text { and } \quad C^{[1 \ldots n+1]}(k)^{\dagger}=C^{[1 \ldots n+1]}(-k)^{t} .
$$

In the same way, one proves recursively that the transmission coefficients $C_{12}^{[1 \ldots n+1]}(k)$ and $C_{21}^{[1 \ldots n+1]}(k)$ depend only on the distances between the different defects, while the reflection coefficients obey

$$
C_{11}^{[1 \ldots n+1]}(k)=\widetilde{C}_{11}^{[1 \ldots n+1]}(k) \mathrm{e}^{-\mathrm{i} k y_{1}} \quad \text { and } \quad C_{22}^{[1 \ldots n+1]}(k)=\widetilde{C}_{22}^{[1 \ldots n+1]}(k) \mathrm{e}^{\mathrm{i} k y_{n+1}},
$$

where the 'bare' coefficients $\widetilde{C}_{j j}^{[1 \ldots n+1]}(k)$ depend only on the distances between the different defects.

\section{Quantum fields with defects}

This section is devoted to some aspects of quantum field theory with defects on the line. In order to keep the discussion as simple as possible we consider one scalar field and $n=2$ defects. The results however can be easily extended to more general cases using the results of section 4 .

\subsection{Correlation functions}

In the Fock representation $\mathcal{F}\left(\mathfrak{A}_{2}\right)$ of $\mathfrak{A}_{2}$ the basic correlation functions are [17]

$$
\left\langle a_{i}(k) a_{j}^{*}(p)\right\rangle=2 \pi\left\{\left[\delta_{i j}+\mathcal{T}_{i j}(k)\right] \delta(k-p)+\mathcal{R}_{i j}(k) \delta(k+p)\right\}, \quad\left\langle a_{i}^{*}(k) a_{j}(p)\right\rangle=0,
$$

where $i, j=1,3$ and $\mathcal{R}$ and $\mathcal{T}$ are given by (3.15+3.20), together with

$$
\mathcal{T}_{11}(k)=\mathcal{T}_{33}(k)=0, \quad \mathcal{R}_{13}(k)=\mathcal{R}_{31}(k)=0 .
$$

Because of (3.9, 3.10), the correlators involving $a_{2}^{*}(k)$ and $a_{2}(k)$ can be expressed in terms of (5.1). Now, using (2.11) one has

$$
\left.\left\langle\varphi\left(t_{1}, x_{1}\right) \varphi\left(t_{2}, x_{2}\right)\right\rangle\right|_{x_{1} \in D_{i}, x_{2} \in D_{j}}=\left\langle\varphi_{i}\left(t_{1}, x_{1}\right) \varphi_{j}\left(t_{2}, x_{2}\right)\right\rangle=\overline{\left\langle\varphi_{j}\left(t_{2}, x_{2}\right) \varphi_{i}\left(t_{1}, x_{1}\right)\right\rangle},
$$

which can be computed for any $i, j=1,2,3$ and have the following general form

$$
\left\langle\varphi_{i}\left(t_{1}, x_{1}\right) \varphi_{j}\left(t_{2}, x_{2}\right)\right\rangle=\int_{-\infty}^{+\infty} \frac{\mathrm{d} k}{4 \pi \omega(k)} \mathrm{e}^{-\mathrm{i} \omega(k) t_{12}}\left\{\left[\delta_{i j}+\mathcal{T}_{i j}(k)\right] \mathrm{e} \mathrm{e}^{\mathrm{i} k x_{12}}+\mathcal{R}_{i j}(k) \mathrm{e}^{\mathrm{i} k \widetilde{x}_{12}}\right\},
$$


where $t_{12}=t_{1}-t_{2}, x_{12}=x_{1}-x_{2}$ and $\widetilde{x}_{12}=x_{1}+x_{2}$. We recall that the dispersion relation is

$$
\omega(k)=\sqrt{k^{2}+m^{2}} .
$$

For the correlators involving $i$ or $j=2$, we use the expressions (3.9, 3.10) and after some algebra we find

$$
\begin{aligned}
& \mathcal{T}_{22}(k)=\frac{\widetilde{R}_{22}^{[1]}(-k) \widetilde{R}_{22}^{[2]}(k) \mathrm{e}^{2 \mathrm{i} k y_{12}}}{1-\widetilde{R}_{22}^{[1]}(-k) \widetilde{R}_{22}^{[2]}(k) \mathrm{e}^{2 \mathrm{i} k y_{12}}}+\frac{\widetilde{R}_{22}^{[1]}(k) \widetilde{R}_{22}^{[2]}(-k) \mathrm{e}^{-2 \mathrm{i} k y_{12}}}{1-\widetilde{R}_{22}^{[1]}(k) \widetilde{R}_{22}^{[2]}(-k) \mathrm{e}^{-2 \mathrm{i} k y_{12}}}, \\
& \mathcal{R}_{22}(k)=\frac{\widetilde{R}_{22}^{[2]}(k) \mathrm{e}^{-2 \mathrm{i} k y_{2}}}{1-\widetilde{R}_{22}^{[1]}(-k) \widetilde{R}_{22}^{[2]}(k) \mathrm{e}^{2 \mathrm{i} k y_{12}}}+\frac{\widetilde{R}_{22}^{[1]}(k) \mathrm{e}^{-2 \mathrm{i} k y_{1}}}{1-\widetilde{R}_{22}^{[1]}(k) \widetilde{R}_{22}^{[2]}(-k) \mathrm{e}^{-2 \mathrm{i} k y_{12}}}, \\
& \mathcal{T}_{12}(k)=\frac{T_{12}^{[1]}(k)}{1-\widetilde{R}_{22}^{[1]}(-k) \widetilde{R}_{22}^{[2]}(k) \mathrm{e}^{2 \mathrm{i} k y_{12}}}, \quad \mathcal{R}_{12}(k)=\frac{T_{12}^{[1]}(k) \widetilde{R}_{22}^{[2]}(k) \mathrm{e}^{-2 \mathrm{i} k y_{2}}}{1-\widetilde{R}_{22}^{[1]}(-k) \widetilde{R}_{22}^{[2]}(k) \mathrm{e}^{2 \mathrm{i} k y_{12}}} \\
& \mathcal{T}_{23}(k)=\frac{T_{23}^{[2]}(k)}{1-\widetilde{R}_{22}^{[1]}(-k) \widetilde{R}_{22}^{[2]}(k) \mathrm{e}^{2 \mathrm{i} k y_{12}}}, \quad \mathcal{R}_{23}(k)=\frac{T_{23}^{[2]}(-k) \widetilde{R}_{22}^{[1]}(k) \mathrm{e}^{-2 \mathrm{i} k y_{1}}}{1-\widetilde{R}_{22}^{[1]}(k) \widetilde{R}_{22}^{[2]}(-k) \mathrm{e}^{-2 \mathrm{i} k y_{12}}},
\end{aligned}
$$

where we have used 'bare' coefficients to make the $y_{j}$ dependence explicit.

The remaining coefficients are computed thanks to (5.3), that is to say

$$
\mathcal{R}_{i j}(k)^{\dagger}=\mathcal{R}_{j i}(-k) \quad \text { and } \quad \mathcal{T}_{i j}(k)^{\dagger}=\mathcal{T}_{j i}(k), \quad i, j=1,2,3
$$

\subsection{Casimir force}

The energy density of the field $\varphi(t, x)$ reads

$$
\theta(t, x)=\frac{1}{2}\left\{\left(\partial_{t} \varphi\right)^{2}-\frac{1}{2}\left[\varphi\left(\partial_{x}^{2} \varphi\right)+\left(\partial_{x}^{2} \varphi\right) \varphi\right]+m^{2} \varphi^{2}\right\}(t, x) .
$$

One can compute the expectation value $\langle\theta(t, x, i)\rangle$ via point-splitting from the two-point function (5.4). Like in the standard Casimir effect, this expectation value diverges at coinciding points. This fact should not be surprising because it is the force, which is actually the physical observable in this context. It is given by

$$
\mathcal{F}_{i}(x)=\left.\frac{\mathrm{d}}{\mathrm{d} x}\langle\theta(t, x)\rangle\right|_{x \in D_{i}}=\mathrm{i} \int_{-\infty}^{+\infty} \frac{\mathrm{d} k}{2 \pi} k \omega(k) \mathcal{R}_{i i}(k) \mathrm{e}^{2 \mathrm{i} k x}, \quad i=1,2,3,
$$

which determines a well-defined distribution in $x$. In general, $\mathcal{F}_{i}(x)$ may be singular at $x=y_{i}$. We will illustrate this feature below on the example of two delta defects. 


\subsection{Finite temperature}

In order to investigate the Casimir force at finite (inverse) temperature $\beta$, we introduce the so called Gibbs representation $\mathcal{G}_{\beta}\left(\mathfrak{A}_{2}\right)$ of $\mathfrak{A}_{2}$. In contrast to the Fock vacuum, the fundamental state of $\mathcal{G}_{\beta}\left(\mathfrak{A}_{2}\right)$ is not annihilated by $a_{i}(k)$ and represents an appropriate

idealization of a thermal bath which keeps the system in equilibrium. In $\mathcal{G}_{\beta}\left(\mathfrak{A}_{2}\right)$ one has $[17]$

$$
\begin{gathered}
\left\langle a_{i}(k) a_{j}^{*}(p)\right\rangle_{\beta}=\frac{1}{1-\mathrm{e}^{-\beta \omega(k)}} 2 \pi\left\{\left[\delta_{i j}+\mathcal{T}_{i j}(k)\right] \delta(k-p)+\mathcal{R}_{i j}(k) \delta(k+p)\right\}, \\
\left\langle a_{i}^{*}(k) a_{j}(p)\right\rangle_{\beta}=\frac{\mathrm{e}^{-\beta \omega(k)}}{1-\mathrm{e}^{-\beta \omega(k)}} 2 \pi\left\{\left[\delta_{i j}+\mathcal{T}_{j i}(k)\right] \delta(k-p)+\mathcal{R}_{j i}(-k) \delta(k+p)\right\},
\end{gathered}
$$

where $i, j=1,3$. It is easy to show at this point that the thermal correlation functions $\left\langle\varphi_{i}\left(t_{1}, x_{1}\right) \varphi_{j}\left(t_{2}, x_{2}\right)\right\rangle_{\beta}$ are obtained by substitution

$$
\mathrm{e}^{-\mathrm{i} \omega(k) t_{12}} \longmapsto \frac{\mathrm{e}^{-\beta \omega(k)+\mathrm{i} \omega(k) t_{12}}+\mathrm{e}^{-\mathrm{i} \omega(k) t_{12}}}{1-\mathrm{e}^{-\beta \omega(k)}}
$$

in the integrand of the respective zero-temperature expressions (5.4). In this way one now gets for the force

$$
\mathcal{F}_{i}(x, \beta)=\mathrm{i} \int_{-\infty}^{\infty} \frac{\mathrm{d} k}{2 \pi} \frac{k \omega(k)}{1-\mathrm{e}^{-\beta \omega(k)}} \mathcal{R}_{i i}(k) \mathrm{e}^{2 \mathrm{i} k x}, \quad i=1,2,3
$$

showing an explicit temperature dependence.

\subsection{Charge density}

In this section we consider a complex scalar field

$$
\varphi(t, x)=\frac{1}{\sqrt{2}}\left[\varphi^{(1)}(t, x)+i \varphi^{(2)}(t, x)\right]
$$

where both $\varphi^{(1)}$ and $\varphi^{(2)}$ are two copies of the scalar field with the same two defects constructed in section 3 above. The theory admits now a $U(1)$ symmetry leading to the conservation of the current

$$
j_{\mu}(t, x)=-i\left[:\left(\partial_{\mu} \varphi^{*}\right) \varphi:(t, x)-: \varphi^{*}\left(\partial_{\mu} \varphi\right):(t, x)\right],
$$

where : $\cdots$ : stands for the normal product in the RT algebra $\mathfrak{A}_{2}$. We are interested in the charge density

$$
\varrho(x, \beta)=\left\langle j_{0}(t, x)\right\rangle_{\beta}
$$


of the system in the Gibbs state, defined in the previous subsection. Using the thermal two-point function of $\varphi$, one obtains

$$
\left.\varrho(x, \beta)\right|_{x \in D_{i}} \equiv \varrho_{i}(x, \beta)=\int_{-\infty}^{\infty} \frac{d k}{\pi} \frac{\mathrm{e}^{-\beta \omega(k)}}{1-\mathrm{e}^{-\beta \omega(k)}}\left(1+\mathcal{R}_{i i}(k) \mathrm{e}^{2 i k x}\right) .
$$

The density (5.19) vanishes in the limit of zero temperature $(\beta \rightarrow \infty)$ : the first term in the integrand gives the density at finite temperature, whereas the second one generates the correction due to the defects.

\section{Example: two delta impurities}

We consider the case of two defects defined by the boundary conditions

$$
\begin{aligned}
& \varphi\left(t,+y_{\alpha}\right)=\varphi\left(t,-y_{\alpha}\right), \\
& \partial_{x} \varphi\left(t,+y_{\alpha}\right)-\partial_{x} \varphi\left(t,-y_{\alpha}\right)=\mu_{\alpha} \varphi\left(t,-y_{\alpha}\right),
\end{aligned} \quad \alpha=1,2, \quad \forall t \in \mathbb{R} .
$$

The local reflection and transmission coefficients have the form

$$
\widetilde{R}_{11}^{[\alpha]}(k)=\widetilde{R}_{22}^{[\alpha]}(-k)=\frac{-i \mu_{\alpha}}{k+i \mu_{\alpha}} \quad \text { and } \quad T_{12}^{[\alpha]}(k)=T_{21}^{[\alpha]}(-k)=\frac{k}{k+i \mu_{\alpha}}, \quad \alpha=1,2 .
$$

To avoid the presence of bound states we will assume that the product $\mu_{1} \mu_{2}$ is strictly positive. In the computation of the Casimir force we will also suppose for simplicity that $m=0$.

\subsection{Force on a test particle between the defects}

Using eq. (5.11), one can compute the force on a test particle when $x \in D_{2}$, that is to say $y_{1}<x<y_{2}$. We get

$$
\begin{aligned}
\pi \mathcal{F}_{2}(x)= & \int_{0}^{+\infty} d k \frac{k^{4}}{k^{4}+G(k)}\left\{k\left[-\mu_{1} \cos \left(2 k\left(x-y_{1}\right)\right)+\mu_{2} \cos \left(2 k\left(x-y_{2}\right)\right)\right]\right. \\
& \left.+\mu_{1}^{2} \sin \left(2 k\left(x-y_{1}\right)\right)+\mu_{2}^{2} \sin \left(2 k\left(x-y_{2}\right)\right)\right\}, \\
G(k)= & k^{2}\left[\left(\mu_{1}+\mu_{2}\right)^{2}-4 \mu_{1} \mu_{2} \sin ^{2}\left(k y_{12}\right)\right]+2 k \mu_{1} \mu_{2}\left(\mu_{1}+\mu_{2}\right) \sin \left(2 k y_{12}\right) \\
& +4 \mu_{1}^{2} \mu_{2}^{2} \sin ^{2}\left(k y_{12}\right) .
\end{aligned}
$$

One can check that the integrand has no real poles, so that the divergent parts are obtain in the limit $k \rightarrow \infty$. They can be computed analytically and we obtain:

$$
\pi \mathcal{F}_{2}(x)=\frac{\mu_{1}}{4\left(x-y_{1}\right)^{2}}-\frac{\mu_{2}}{4\left(x-y_{2}\right)^{2}}+\frac{\mu_{1}^{2}}{2\left(x-y_{1}\right)}-\mathfrak{c}_{2}\left(x, y_{1}, y_{2}\right)-\mathfrak{s}_{2}\left(x, y_{1}, y_{2}\right)
$$


where we have introduced

$$
\begin{aligned}
\mathfrak{c}_{2}\left(x, y_{1}, y_{2}\right) & =\int_{0}^{+\infty} d k \frac{k G(k)}{k^{4}+G(k)}\left\{-\mu_{1} \cos \left[2 k\left(x-y_{1}\right)\right]+\mu_{2} \cos \left[2 k\left(x-y_{2}\right)\right]\right\}, \\
\mathfrak{s}_{2}\left(x, y_{1}, y_{2}\right) & =\int_{0}^{+\infty} d k \frac{G(k)}{k^{4}+G(k)}\left\{\mu_{1}^{2} \sin \left[2 k\left(x-y_{1}\right)\right]+\mu_{2}^{2} \sin \left[2 k\left(x-y_{2}\right)\right]\right\} .
\end{aligned}
$$

The integral $\mathfrak{c}_{2}\left(x, y_{1}, y_{2}\right)$ has logarithmic singularities at $x=y_{1}, y_{2}, x=2 y_{1}-y_{2}$ and $x=2 y_{2}-y_{1}$, which can be extracted by means of the Meijer's function

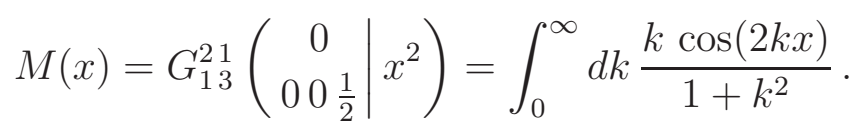

We get

$$
\begin{aligned}
\mathfrak{c}_{2}\left(x, y_{1}, y_{2}\right)= & -\mu_{1}^{3} M\left(x-y_{1}\right)+\mu_{2}^{3} M\left(x-y_{2}\right)-\mu_{1}^{2} \mu_{2} M\left(x+y_{2}-2 y_{1}\right) \\
& +\mu_{1} \mu_{2}^{2} M\left(x+y_{1}-2 y_{2}\right)+\widetilde{\mathfrak{c}}_{2}\left(x, y_{1}, y_{2}\right)
\end{aligned}
$$

The integrals $\widetilde{\mathfrak{c}}_{2}\left(x, y_{1}, y_{2}\right)$ and $\mathfrak{s}_{2}\left(x, y_{1}, y_{2}\right)$ are absolutely convergent and can be computed numerically. We represent the force for different values of $\mu_{1}$ and $\mu_{2}$ in figures 1 and 2 .

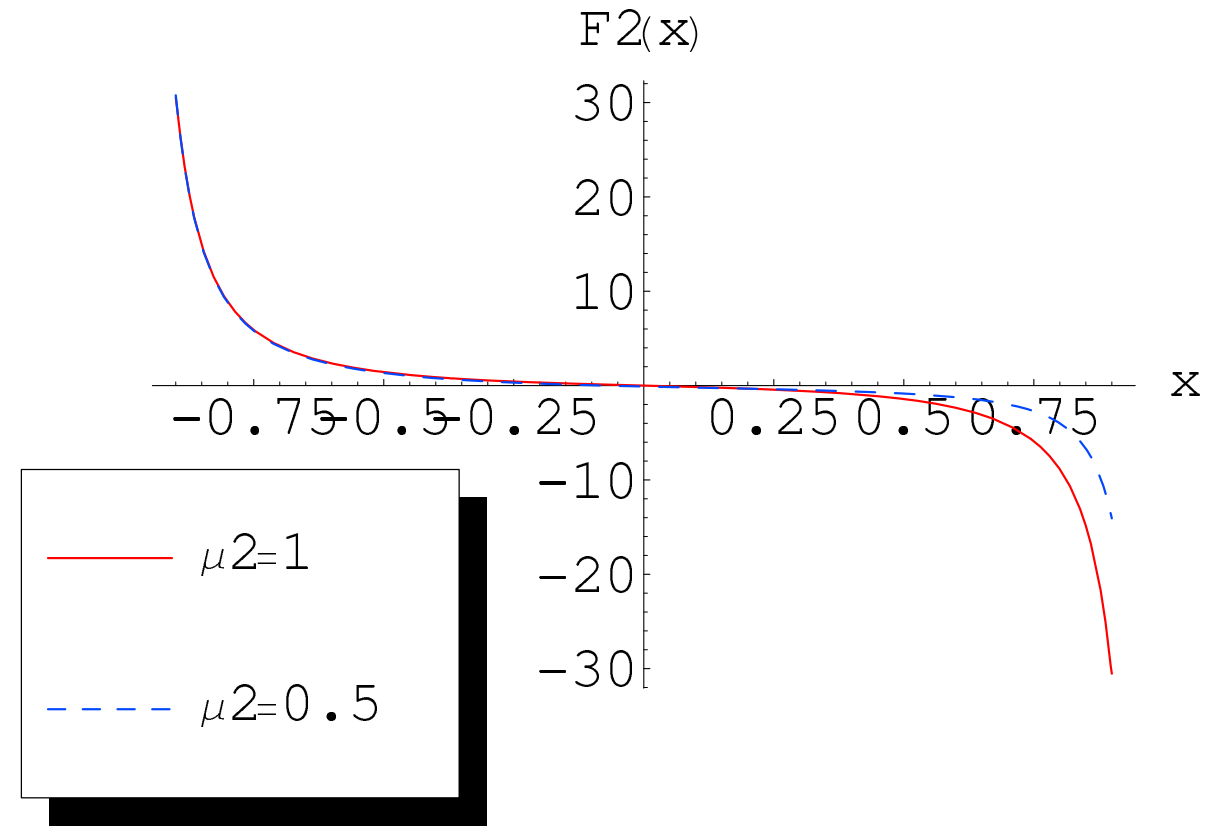

Figure 1: Force as a function of the position of the test particle $x \in] y_{1}, y_{2}$ [ for two delta impurities, with $y_{2}=-y_{1}=1$ and $\mu_{1}=1$.

We find a point between the defects where the force changes sign. In this point the test particle is in stable equilibrium. 


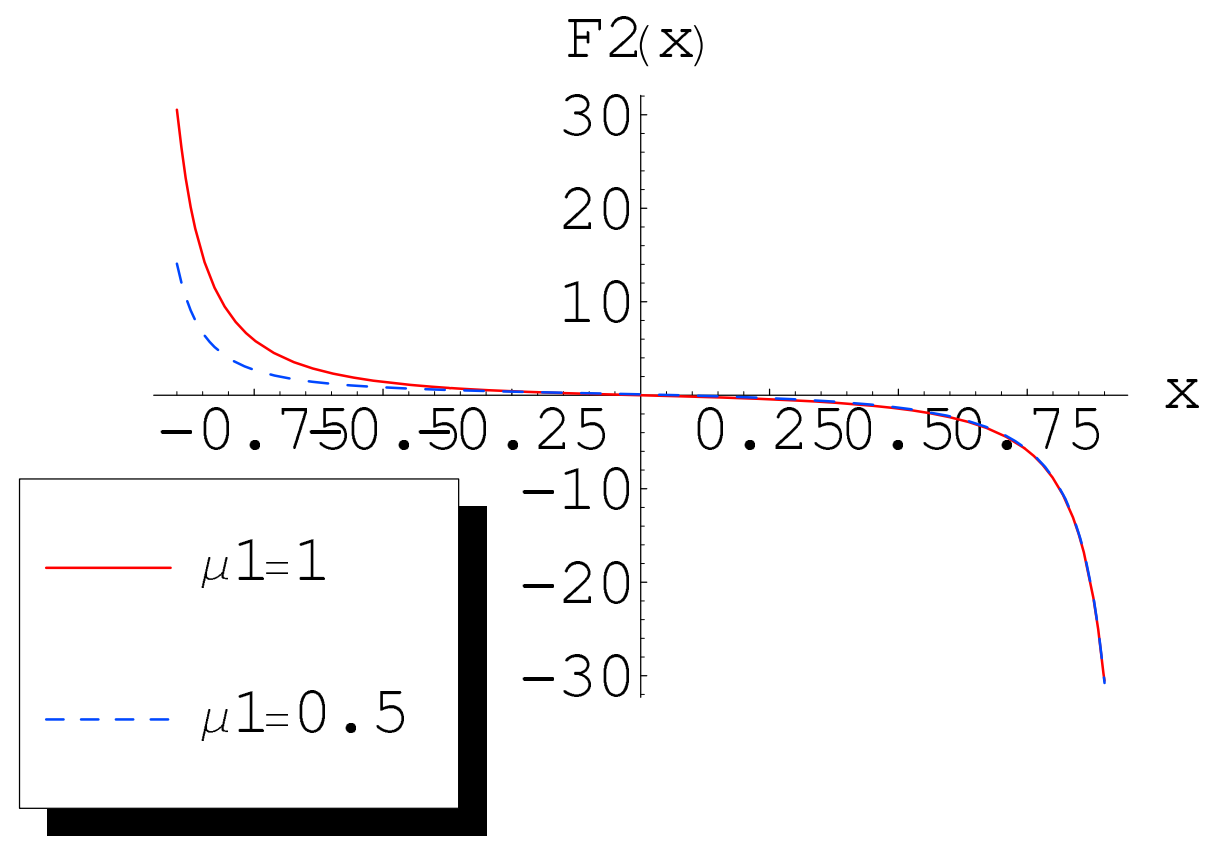

Figure 2: Force as a function of the position of the test particle $x \in] y_{1}, y_{2}$ [ for two delta impurities, with $y_{2}=-y_{1}=1$ and $\mu_{2}=1$.

\subsection{Force on a test particle on the left of the defects}

We perform the same kind of calculation when $x \in D_{1}$, that is to say $x<y_{1}$. Extracting the poles, we get

$$
\begin{aligned}
\pi \mathcal{F}_{1}(x)= & \frac{-\mu_{1}}{4\left(x-y_{1}\right)^{2}}-\frac{\mu_{2}}{4\left(x-y_{2}\right)^{2}}+\frac{\mu_{1}^{2}}{2\left(x-y_{1}\right)}+\frac{\mu_{2}^{2}+2 \mu_{1} \mu_{2}}{2\left(x-y_{2}\right)} \\
& -\mathfrak{c}_{1}\left(x, y_{1}, y_{2}\right)-\mathfrak{s}_{1}\left(x, y_{1}, y_{2}\right)+\int_{0}^{+\infty} d k \frac{k \mathfrak{a}_{1}(k)+\mathfrak{a}_{0}(k)}{k^{4}+G(k)}
\end{aligned}
$$

where we have introduced:

$$
\begin{aligned}
& \mathfrak{c}_{1}\left(x, y_{1}, y_{2}\right)=\int_{0}^{+\infty} d k \frac{k G(k)}{k^{4}+G(k)}\left\{\mu_{1} \cos \left[2 k\left(x-y_{1}\right)\right]+\mu_{2} \cos \left[2 k\left(x-y_{2}\right)\right]\right\}, \\
& \mathfrak{s}_{1}\left(x, y_{1}, y_{2}\right)=\int_{0}^{+\infty} d k \frac{G(k)}{k^{4}+G(k)}\left\{\mu_{1}^{2} \sin \left[2 k\left(x-y_{1}\right)\right]+\left(\mu_{2}^{2}+2 \mu_{1} \mu_{2}\right) \sin \left[2 k\left(x-y_{2}\right)\right]\right\}, \\
& \mathfrak{a}_{1}(k)=2 \mu_{1} \mu_{2}\left\{\mu_{2} \cos \left[2 k\left(x-y_{1}\right)\right]-\mu_{2} \cos \left[2 k\left(x-y_{2}\right)\right]+\mu_{1} \sin \left[2 k\left(x-y_{1}\right)\right] \sin \left(2 k y_{12}\right)\right\}, \\
& \mathfrak{a}_{0}(k)=2 \mu_{1}^{2} \mu_{2}^{2} \sin \left[2 k\left(x-y_{1}\right)\right]\left[1-\cos \left(2 k y_{12}\right)\right] .
\end{aligned}
$$


Again, one can extract from $\mathfrak{c}_{1}\left(x, y_{1}, y_{2}\right)$ the Meijer's contribution

$$
\begin{aligned}
\mathfrak{c}_{1}\left(x, y_{1}, y_{2}\right)= & -\mu_{1}^{3} M\left(x-y_{1}\right)+\mu_{2}\left(\left(\mu_{1}+\mu_{2}\right)^{2}+2 \mu_{1}^{2}\right) M\left(x-y_{2}\right) \\
& -\mu_{1} \mu_{2}^{2} M\left(x+y_{1}-2 y_{2}\right)+\widetilde{\mathfrak{c}}_{1}\left(x, y_{1}, y_{2}\right) .
\end{aligned}
$$

The remaining integrals are absolutely convergent and can be computed numerically. We represent the force in figures 3 and 4 , which show that it is repulsive close to the defect at $y_{1}$, but for $x<y_{1}$ there are also domains of attraction.

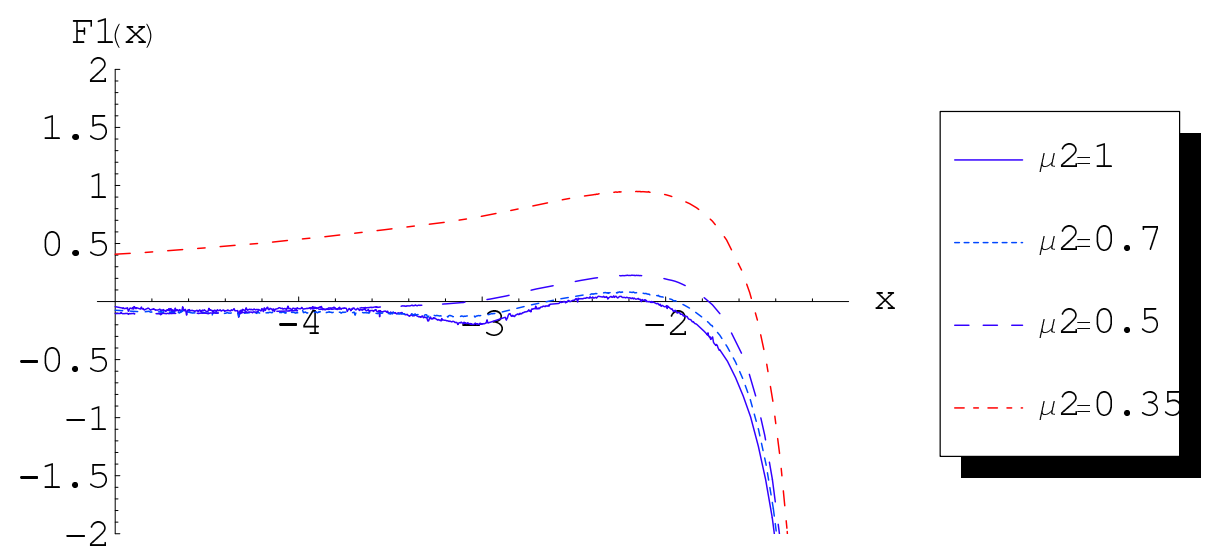

Figure 3: Force as a function of the position of the test particle $x<y_{1}$ for two delta defects, with $y_{2}=-y_{1}=1$ and $\mu_{1}=1$.

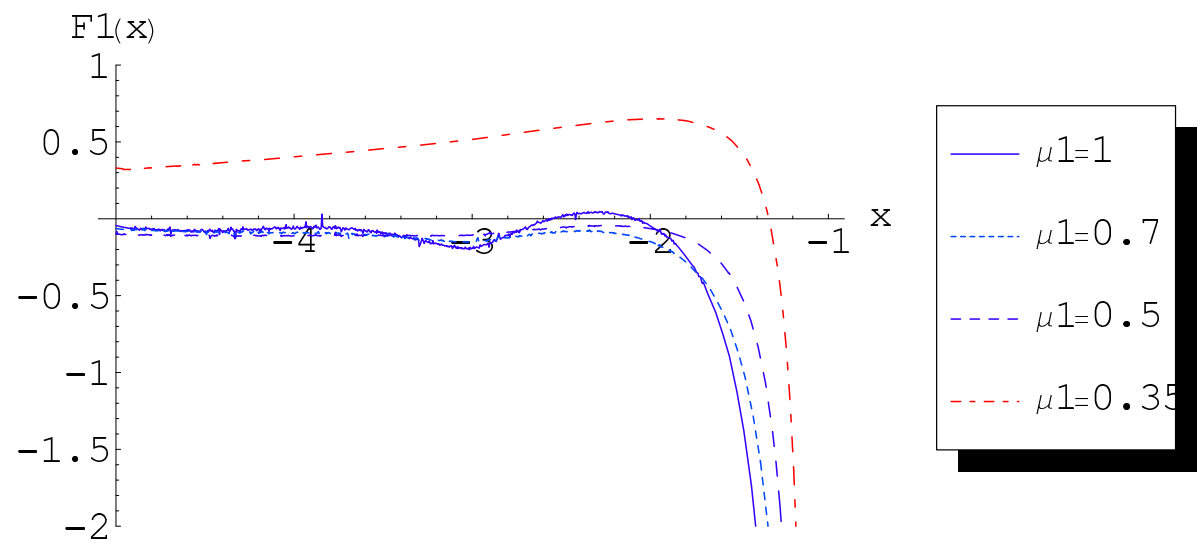

Figure 4: Force as a function of the position of the test particle $x<y_{1}$ for two delta defects, with $y_{2}=-y_{1}=1$ and $\mu_{2}=1$. 


\subsection{Force at the defect}

As already mentioned, the force is a distribution with singularities at the positions $x=$ $y_{1}, y_{2}$ of the defects. To compute the force acting for instance at the defect in $y_{1}$, one needs to sum the two contributions computed in sections 6.1 and 6.2 and take the limit $x \rightarrow y_{1}$, getting rid of the divergences. This is achieved by taking the symmetric limit:

$$
\mathcal{F}_{12}=\lim _{\delta \rightarrow 0^{+}}\left(\mathcal{F}_{1}\left(y_{1}-\delta\right)+\mathcal{F}_{2}\left(y_{1}+\delta\right)\right) \text {. }
$$

One obtains the force as a function of the distance between the two defects:

$$
\begin{aligned}
\pi \mathcal{F}_{12}= & -\frac{\mu_{2}}{2 y_{12}^{2}}+\frac{\mu_{2}\left(\mu_{2}+\mu_{1}\right)}{y_{12}}-2 \mu_{2}\left(\mu_{1}+\mu_{2}\right) \int_{0}^{+\infty} d k \frac{G(k)}{k^{4}+G(k)} \sin \left(2 k y_{12}\right) \\
& -2 \mu_{1} \mu_{2}^{2} \int_{0}^{+\infty} \frac{k d k}{k^{4}+G(k)}\left[\left(\mu_{1}+\mu_{2}\right) k \sin \left(4 y_{12}\right)+4 \mu_{1} \mu_{2} \cos \left(2 k y_{12}\right) \sin ^{2}\left(k y_{12}\right)\right] \\
& -2 \mu_{2} \int_{0}^{+\infty} d k \frac{k^{3}}{k^{4}+G(k)}\left[\mu_{1} \mu_{2} \cos \left(4 k y_{12}\right)+\left(\mu_{1}^{2}+\mu_{2}^{2}+\mu_{1} \mu_{2}\right) \cos \left(2 k y_{12}\right)\right] .
\end{aligned}
$$

Using the Meijer's function, one finds for the last integral

$$
-2 \mu_{1} \mu_{2}^{2} M\left(2 y_{12}\right)-2 \mu_{2}\left(\mu_{1}^{2}+\mu_{2}^{2}+\mu_{1} \mu_{2}\right) M\left(y_{12}\right)+\mathfrak{c}_{12}\left(y_{12}, \mu_{1}, \mu_{2}\right)
$$

where $\mathfrak{c}_{12}\left(y_{12}, \mu_{1}, \mu_{2}\right)$ is absolutely convergent. Computing the integrals numerically, one gets the figures 5 and 6 .

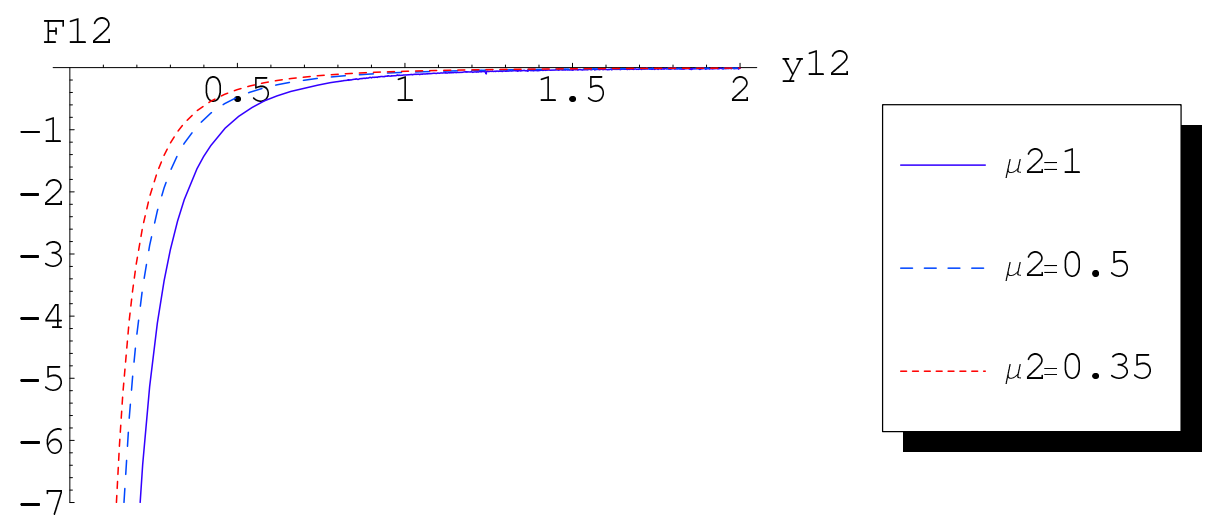

Figure 5: Force at the defect for two delta defects, as a function of the distance between them for $\mu_{1}=1$. 


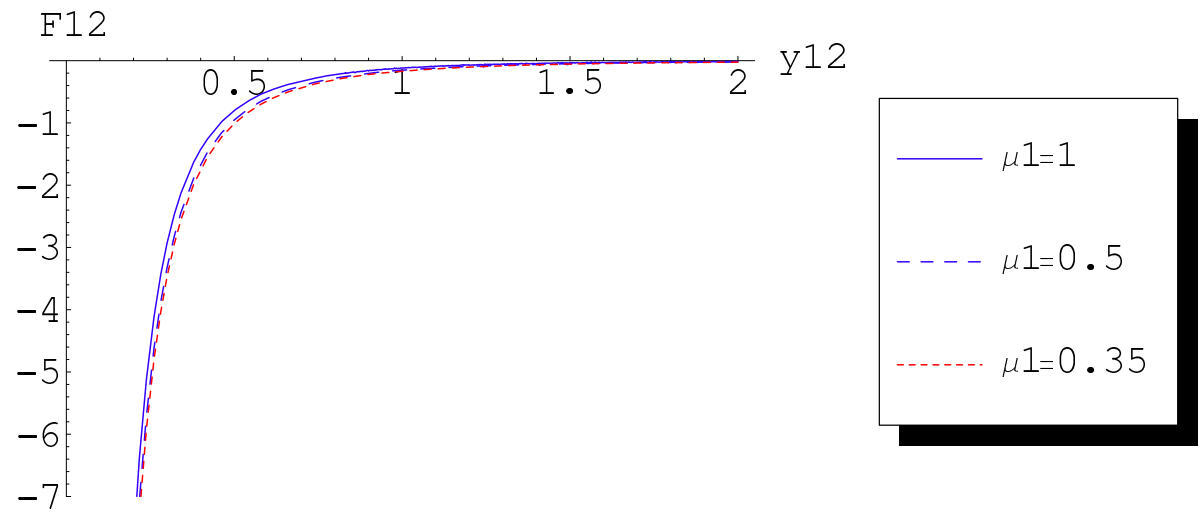

Figure 6: Force at the defect for two delta defects, as a function of the distance between them for $\mu_{2}=1$.

\subsection{Charge density}

The charged density for a complex field and two delta-defects is obtained from (5.19). The integrals can be computed numerically, the mass $m$ and the temperature $\frac{1}{\beta}$ playing the role of regulators at $k=0$ and $k=\infty$ respectively. We get

$$
\widehat{\rho}_{j}(x, \beta)=\rho_{j}(x, \beta)-\int_{-\infty}^{\infty} \frac{d k}{\pi} \frac{\mathrm{e}^{-\beta \omega(k)}}{1-\mathrm{e}^{-\beta \omega(k)}}=\int_{-\infty}^{\infty} \frac{d k}{\pi} \frac{\mathrm{e}^{-\beta \omega(k)}}{1-\mathrm{e}^{-\beta \omega(k)}} \frac{\mathfrak{r}_{j}(x)}{k^{4}+G(k)}, \quad j=1,2,
$$

with

$$
\begin{aligned}
\mathfrak{r}_{2}(k)= & 2\left\{\mu_{1} \sin \left[2 k\left(x-y_{1}\right)\right]-k \mu_{2} \sin \left[2 k\left(x-y_{2}\right)\right]\right\} k^{3} \\
& +2\left\{\mu_{1}^{2} \cos \left[2 k\left(x-y_{1}\right)\right]+\mu_{2}^{2} \cos \left[2 k\left(x-y_{2}\right)\right]\right\} k^{2}, \\
\mathfrak{r}_{1}(x)= & 2\left\{\mu_{1} \sin \left[2 k\left(x-y_{1}\right)\right]+\mu_{2} \sin \left[2 k\left(x-y_{2}\right)\right]\right\} k^{3} \\
& -2\left\{\mu_{1}^{2} \cos \left[2 k\left(x-y_{1}\right)\right]+\mu_{2}\left(2 \mu_{1}+\mu_{2}\right) \cos \left[2 k\left(x-y_{2}\right)\right]\right\} k^{2} \\
& -4 \mu_{1} \mu_{2} \sin \left(2 k y_{12}\right)\left\{\mu_{1} \cos \left[2 k\left(x-y_{1}\right)\right]+\mu_{2} \cos \left[k\left(2 x-y_{1}-y_{2}\right)\right]\right\} k \\
& -4 \mu_{1}^{2} \mu_{2}^{2}\left[1-\cos \left(2 k y_{12}\right)\right] \cos \left[2 k\left(x-y_{1}\right)\right] .
\end{aligned}
$$

and a similar expression for $\mathfrak{r}_{3}(k)$. We remind that $\omega(k)=\sqrt{k^{2}+m^{2}}$.

When $m=0$, there is a divergence at $k=0$ reflecting the possibility of Bose condensation. When $m \neq 0$ (and $\beta \neq 0$ ), the integral defining $\rho_{j}(x, \beta), j=1$ or 3 , converges. The same is true for $\rho_{2}(x, \beta)$ and generic values of the parameters $\mu_{1}, \mu_{2}, y_{1}$ and $y_{2}$. However, when the relation

$$
\mu_{1}+\mu_{2}=2 \mu_{1} \mu_{2}\left(y_{2}-y_{1}\right)
$$


is fulfilled, $\rho_{2}(x, \beta)$ diverges (see figure 7). This relation corresponds to a change in the behavior of $G(k)$ around $k=0$ :

$$
G(k) \underset{k=0}{\sim}\left(\mu_{1}+\mu_{2}-2 \mu_{1} \mu_{2} y_{21}\right)^{2} k^{2}+o\left(k^{4}\right)
$$

and could be interpreted as a kind of resonance effect. One can check that, at this point in the parameter space, the integrals entering in $\rho_{j}(x, \beta), j=1$ or 3 , as well as in the force studied in the previous sections, still converge.

The plots of $\widehat{\rho}_{j}(x, \beta)$ are presented in figures 8,10 ,

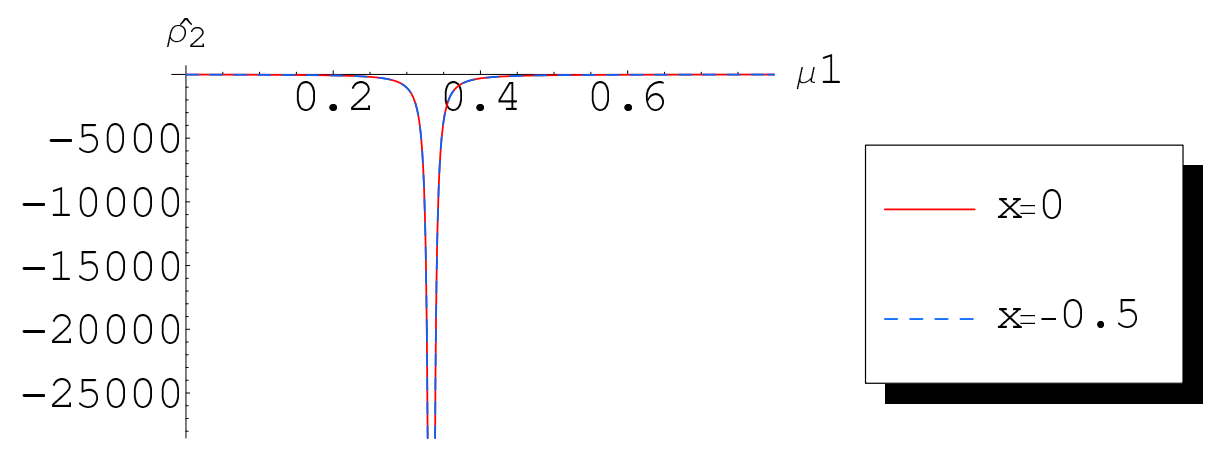

Figure 7: Density between two delta defects, as a function of $\mu_{1}$ for $\mu_{2}=1, y_{2}=1=-y_{1}$ and $\beta=1$.

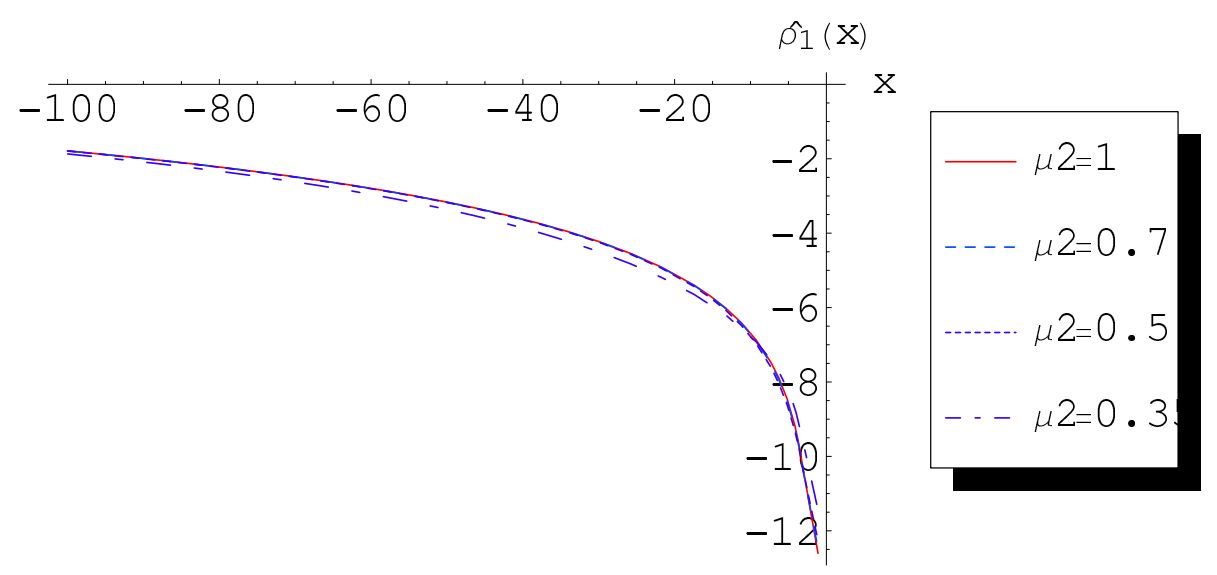

Figure 8: Density on the left of two delta defects, as a function of $x$ for $\mu_{1}=1, y_{2}=1=$ $-y_{1}$ and $\beta=1$ (large distance behavior). 


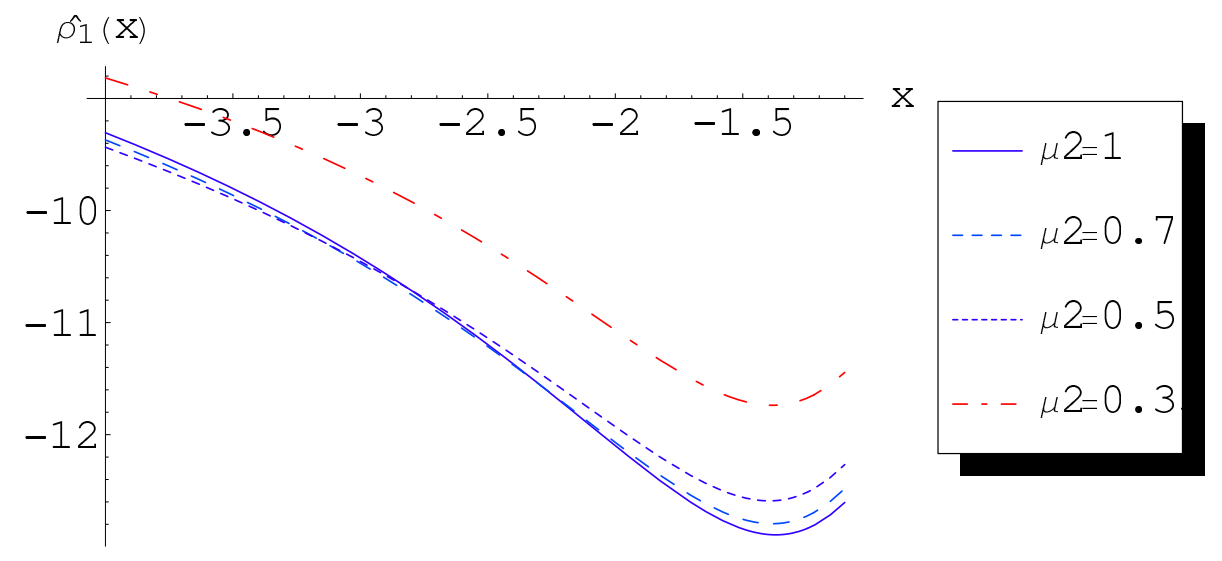

Figure 9: Density on the left of two delta defects, as a function of $x$ for $\mu_{1}=1, y_{2}=1=$ $-y_{1}$ and $\beta=1$ (zoom of figure 8, close to the defect in $y 1=-1$ ). 

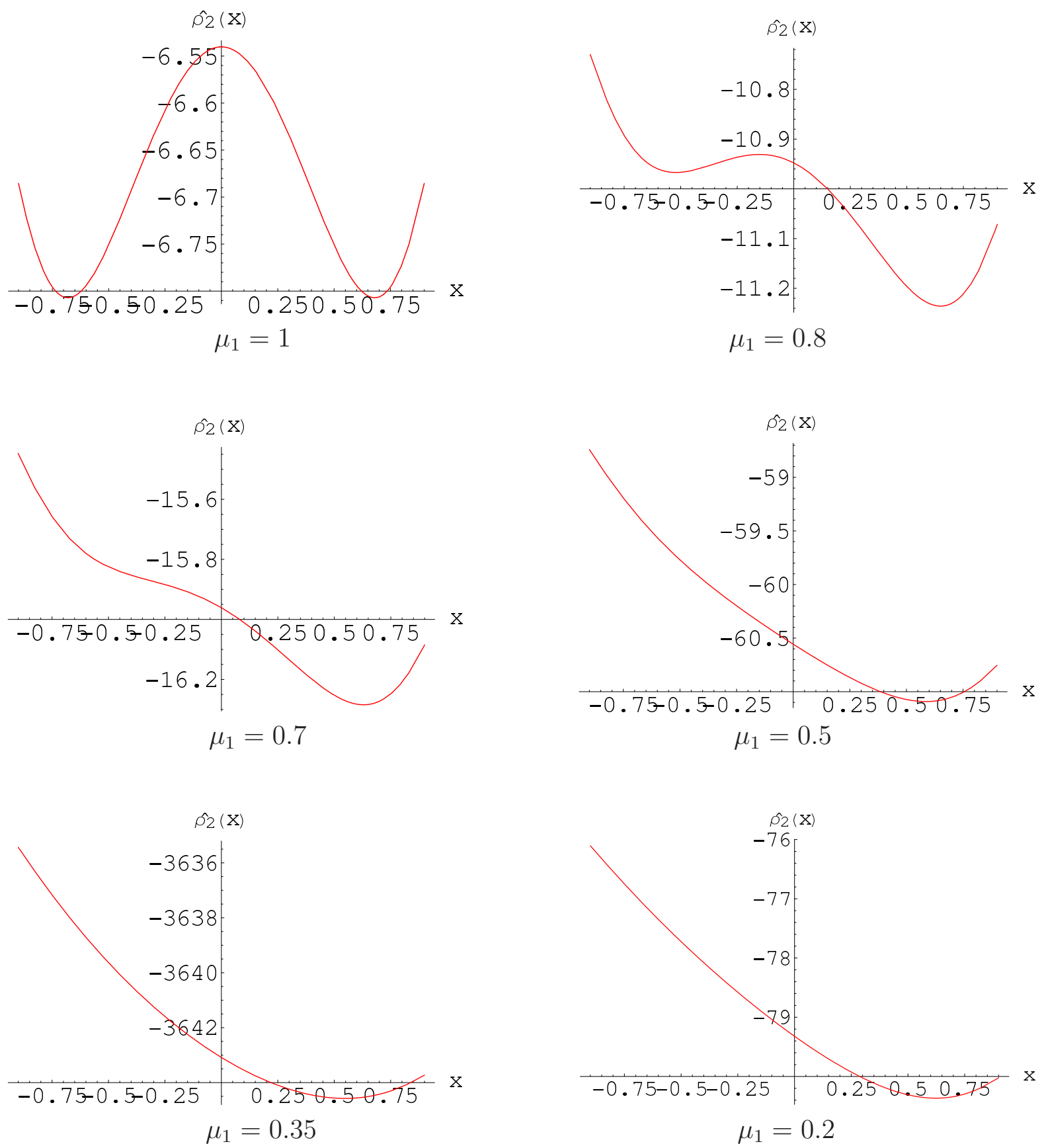

Figure 10: Density between two delta defects, as a function of $x$ for $\mu_{2}=1$ and $\beta=1$. 


\section{Conclusion}

In the present paper we generalized the RT algebra framework [14]- [16] for dealing with one defect to any number of defects. More precisely, we considered a scalar field, which interacts with multiple defects on the line and freely propagates away of them. We studied the most general point-like defects, parametrizing the interaction by local reflection and transmission coefficients. As expected, the algebraic formulation considerably simplifies the boundary value problem at hand and is very efficient for deriving the correlation functions both at zero and finite temperature. Applied to the Casimir effect, the algebraic technique leads to a nice and compact expression (in terms of the reflection coefficients) for the force. As an explicit illustration, we derived the Casimir force for two delta-type defects. It turns out that both the intensity and the direction of the force depend on the position. We established also the charge density distribution of a complex scalar field interacting with the defects.

Our framework can be generalized in several directions. One can apply it to the case where the field $\varphi$ has $N>1$ internal degrees of freedom. Then, each of the operators

$a_{i}(k), i=1, \ldots, n$, becomes a vector of length $N$, while the 'coefficients' $C_{i j}^{[\alpha]}, i, j=1,2$ are now $N \times N$ matrices. The above results remain valid if these matrices commute among them.

Another very attractive application concerns quantum graphs [21,22], which are networks of wires connected at nodes. Each node is characterized by a scattering matrix [23] and can be viewed as a defect [24]. The RT algebra approach has been already applied [25] to the study of quantum fields on graphs with one vertex (star graphs). Using the results of this paper, one can formulate quantum field theory on a generic quantum graph and address in this framework the interesting problems of conductance and vacuum energy.

\section{Acknowledgments}

E.R. warmly thanks E. Pilon and JP. Guillet for useful advice on the analytical and numerical (resp.) estimates of the integrals computed in section 6 .

\section{References}

[1] C. L. Kane and M. P. A. Fisher, Phys. Rev. B 46 (1992) 15233.

[2] C. Nayak, M. P. A. Fisher, A. W. W. Ludwig and H. H. Lin, Phys. Rev. B 59 (1999) 15694.

[3] X. Barnabe-Theriault, A. Sedeki, V. Meden, K. Schönhammer Phys. Rev. B 71 (2005) 205327. 
[4] M. Oshikawa, C. Chamon and I. Affleck, J. Stat. Mech. 0602 (2006) P008 arXiv:cond-mat/0509675.

[5] E. Fradkin and J. E. Moore, Phys. Rev. Lett. 97 (2006) 050404 arXiv:cond-mat/0605683.

[6] H. Saleur, "Lectures on Non-perturbative field theory and quantum impurity problems", arXiv:cond-mat/9812110.

[7] H. Saleur, "Lectures on Non-perturbative field theory and quantum impurity problems II", arXiv:cond-mat/0007309.

[8] I. Cherednik, Int. J. Mod. Phys. A 7 (1992) 109.

[9] G. Delfino, G. Mussardo and P. Simonetti, Nucl. Phys. B 432 (1994) 518 arXiv:hep-th/9409076].

[10] R. Konik and A. LeClair, Nucl. Phys. B 538 (1999) 587 [arXiv:hep-th/9703085].

[11] O. Castro-Alvaredo and A. Fring, Nucl. Phys. B 649 (2003) 449 arXiv:hep-th/0205076.

[12] P. Bowcock, E. Corrigan and C. Zambon, Int. J. Mod. Phys. A 19S2 (2004) 82 arXiv:hep-th/0305022.

[13] P. Bowcock, E. Corrigan and C. Zambon, JHEP $0401 \quad$ (2004) 056 arXiv:hep-th/0401020].

[14] M. Mintchev, E. Ragoucy and P. Sorba, Phys. Lett. B 547 (2002) 313. arXiv:hep-th/0209052.

[15] M. Mintchev, E. Ragoucy and P. Sorba, J. Phys. A 36 (2003) 10407 arXiv:hep-th/0303187.

[16] V. Caudrelier, M. Mintchev, E. Ragoucy and P. Sorba, J. Phys. A 38 (2005) 3431 arXiv:hep-th/0412159.

[17] M. Mintchev and P. Sorba, JSTAT 0407 (2004) P001 arXiv:hep-th/0405264].

[18] M. Mintchev and P. Sorba, Annales Henri Poincare 7 (2006) 1375 arXiv:hep-th/0511162.

[19] S. Albeverio, L. Dabrowski and P. Kurasov, Lett. Math. Phys. 45 (1998) 33.

[20] A. G. M. Schmidt, B. K. Cheng and M. G. E. da Luz, Phys. Rev. A 66 (2002) 062712.

[21] P. Kuchment, Waves Random Media 12 (2002) R1. 
[22] P. Kuchment, Waves Random Media 14 (2004) S107.

[23] V. Kostrykin and R. Schrader, J. Phys. A 32 (1999) 595.

[24] B. Bellazzini and M. Mintchev, J. Phys. A 39 (2006) 11101 arXiv:hep-th/0605036].

[25] B. Bellazzini, M. Mintchev and P. Sorba, J. Phys. A 40 (2007) 2485 arXiv:hep-th/0611090. 